\title{
碳纳米管/聚苯胺化学修饰电极的制备及其对抗坏血酸的检测
}

\author{
邓敏, 江 奇, 方渊, 李 欢, 邱家欣, 卢晓英
}

(西南交通大学 超导与新能源研发中心, 生命科学与工程学院, 材料先进技术教育部重点实验室, 成都 610031)

摘 要: 通过恒电压沉积法将纳米金属镍沉积于石墨电极表面, 经化学气相沉积法在石墨电极表面原位生长出碳纳 米管(CNTs), 通过电化学聚合法在 CNTs 表面原位聚合聚苯胺, 从而获得化学修饰电极。采用扫描电子显微镜对所 得电极形貌结构进行表征, 并研究 CNTs 与 PNAI 复合电极对抗坏血酸(AA)的检测效果。研究结果表明: 制备的 CNTs 都能均匀地生长在石墨电极表面, 纳米中空管状结构都保持完好; PANI 均匀地包覆在 CNTs 管壁上, 复合材 料呈现出典型的三维网状结构。所制备的 CNTs/PANI 修饰电极对 AA 具有良好的电化学响应, 其中管径较小 CNTs 的修饰电极对 AA 的电化学响应更强：具有更宽的检测范围和更低的检出限。其检测线性范围为 $1.0 \times 10^{-6} \sim 4.5 \times 10^{-4} \mathrm{~mol} / \mathrm{L}$, 检出限为 $1.0 \times 10^{-7} \mathrm{~mol} / \mathrm{L}(\mathrm{S} / \mathrm{N}=3)$ 。且具有良好的稳定性、重复性和可靠性。

关 键 词: 抗坏血酸; 碳纳米管/聚苯胺; 化学修饰电极; 电化学检测; 碳纳米管管径

中图分类号: 0657 文献标识码: A

\section{Carbon Nanotubes/Polyaniline Chemically Modified Electrode: Preparation and Ascorbic Acid Detection}

\author{
DENG Min, JIANG Qi, FANG Yuan, LI Huan, QIU Jia-Xin, LU Xiao-Ying
}

(Key Laboratory of Advanced Technologies of Materials (Ministry of Education of China), Superconductivity and New Energy R\&D Centre, School of Life Science and Engineering, Southwest Jiaotong University, Chengdu 610031, China)

\begin{abstract}
Nickel catalyst was deposited on a graphite electrode (GE) surface by constant voltage deposition method. With the nickel catalyst, carbon nanotubes (CNTs) were grown in situ on the GE surface to prepare CNTs chemically modified electrode (GSCNTs-CME) by catalytic chemical vapor deposition. After that, polyaniline (PANI) was polymerized in situ on the GSCNTs-CME to obtain GSCNTs/PANI-CME by electrochemical polymerization. Morphology and structure of the obtained electrodes were characterized by scanning electron microscope. Detection performances of the GSCNTs/PANI-CME on ascorbic acid (AA) were evaluated on an electrochemical workstation. Results show that the CNTs grow uniformly on the GE surface and the original tubular structure is remained well. PANI is coated uniformly on the surface of CNTs in the obtained composite with a typical three-dimensional network structure. The GSCNTs/PANI-CMEs show excellent electrochemical response to AA, amongst which the GSCNTs/PANI-CME with small-diameter CNTs shows stronger electrochemical response (wider linear detection range and lower detection limit) to AA. And its linear detection range and detection limit are
\end{abstract}

收稿日期: 2017-04-20；收到修改稿日期：2017-05-27

基金项目：国家自然科学基金(50907056，51602266);四川省重点研发计划(2017GZ0109); 四川省科技支撑项目(2016GZ0273, 2016GZ0275); 四川省学术与技术带头人培养基金; 四川省成都市科技惠民工程(2014-HM01-00073-SF) National Natural Science Foundation of China (50907056, 51602266); Sichuan Key Research and Development Program (2017GZ0109); Sichuan Science and Technology Support Projects (2016GZ0273, 2016GZ0275); Sichuan Academic and Technical Leaders Training Fund; Chengdu Science and Technology Huimin Project (2014-HM0100073-SF)

作者简介: 邓 敏(1991-), 女, 硕士研究生. E-mail: 3105a@swjtu.cn

通讯作者: 江 奇, 教授. E-mail: jiangqi66@163.com 
$1.0 \times 10^{-6} \sim 4.5 \times 10^{-4} \mathrm{~mol} / \mathrm{L}$ and $1.0 \times 10^{-7} \mathrm{~mol} / \mathrm{L}(\mathrm{S} / \mathrm{N}=3)$, respectively. Therefore, the GSCNTs/PANI-CME shows excellent stability, repeatability and reliability.

Key words: ascorbic acid; carbon nanotubes/polyaniline; chemically modified electrode; electrochemical detection; carbon nanotube diameter

抗坏血酸(Ascorbic Acid, AA) 又称维生素 C, 是 一种有效的还原剂和抗氧化剂, 并广泛存在于食物 和人体器官中 ${ }^{[1]}$ 。发展一种简便、快速、精确检测 AA 的方法对于食物和药品安全十分重要。比起传 统的 $\mathrm{AA}$ 检测方法, 如苂光法、色谱法、分光光度 法等, 电化学方法具有造价低、灵敏度高、反应速 度快和操作简单等优点 ${ }^{[2-3]}$ 。化学修饰电极(Chemically Modified Electrode, CME)因具有良好的选择性和灵 敏性, 在 AA 的检测中备受关注 ${ }^{[4-8]}$ 。

聚苯胺(Polyaniline, PANI)因对 AA 具有较高的 电催化性和选择性而受到关注, 但较弱的导电性和 稳定性又制约了其应用 ${ }^{[9-10]}$ 。碳纳米管 (Carbon Nanotubes, CNTs)自首次被报道以来 ${ }^{[11]}$, 因高比表 面积、强吸附能力, 高导电性和良好化学稳定性, 在 电化学传感器领域受到极大关注 ${ }^{[12-15]}$ 。因此 PANI 和 CNTs 之间存在结构与性能上强的互补性, 基于 PANI 和 CNTs 复合材料的 CME 也陆续被报道, 并 展示出优良的电化学性能 ${ }^{[16-19]}$ 。

目前 CNTs/PANI-CME 可采用以下方法制备： (1)先将功能化的 CNTs 通过滴加或吸附固定在裸电 极表面, 然后 PANI 通过化学聚合或电聚合在 CNTs 表面 ${ }^{[20-23] ;}$; (2)先制备 CNTs/PANI 复合材料, 再通过 滴加或吸附将复合材料固定在电极表面 ${ }^{[24-27]}$ 。为了 增强电极材料与电极表面的结合力, 上述两种方法 往往会使用交联剂, 但交联剂的加入会破坏 CNTs 原有的纳米空心管结构, 增加体系电阻, 导致 CNTs 利用率下降, 从而影响修饰材料性能的发挥。因此, 本工作提出一种新型的 CNTs /PANI-CME 原位生长 (Grown in situ, GS) 方法, 在整个电极制备过程不添 加交联剂。

\section{1 实验方法}

\section{1 电化学沉积催化剂镍}

将圆形柱状 $(\phi=5.3 \mathrm{~mm}, l=7.0 \mathrm{~mm}$ )石墨电极 $(\mathrm{GE})$ 的圆形表面打磨平整, 抛光后装入聚四氟乙烯电极 套中, 用硅胶密封后作为工作电极。为了制备出不 同晶粒大小的镍颗粒, 分别以恒电位 $1 \mathrm{~V}$ 和 $2.05 \mathrm{~V}$ 在镀液(每 $50 \mathrm{~mL}$ 溶液中含硫酸镍 $15.00 \mathrm{~g}$, 氯化镍 $2.00 \mathrm{~g}$, 硼酸 $2.00 \mathrm{~g}$, 十二烷基硫酸钠 $0.05 \mathrm{~g}$, 糖精
钠 $0.02 \mathrm{~g}$ )中进行直流电化学沉积 $5 \mathrm{~min}$ 。然后从电 极套中取出石墨电极, 用去离子水冲洗, 室温下干燥。

\subsection{GSCNTs-CME 的制备}

在管式电阻炉中利用化学气相沉积法制备 GSCNTs-CME ${ }^{[28-29]}$ : 将预处理后的石墨电极放入石 英舟中, 然后将石英舟送入到石英管 $(\phi=60 \mathrm{~mm}$, $l=1000 \mathrm{~mm})$ 中部恒温区。先通入流速为 $50 \mathrm{sccm}$ 的 氩气(Ar) 以排出石英管中的空气，同时管式炉以 $10^{\circ} \mathrm{C} / \mathrm{min}$ 的速率从室温加热到 $800^{\circ} \mathrm{C}$, 达到 $800^{\circ} \mathrm{C}$ 后 通入 $\mathrm{H}_{2}(50 \mathrm{sccm})$ 还原 $30 \mathrm{~min}$, 然后在 $\operatorname{Ar}(50 \mathrm{sccm})$ 保护下降温到 $700^{\circ} \mathrm{C}$ 再通入 $\mathrm{C}_{2} \mathrm{H}_{2}$ 和 $\mathrm{Ar}$ 的混合气体 $\left(\mathrm{C}_{2} \mathrm{H}_{2} / \mathrm{Ar}, 20 / 160\right)$ 反应 $20 \mathrm{~min}$, 最后在氩气 $(25 \mathrm{sccm})$ 保护下冷却至室温。从石英管中取出电极, 将电极 放入浓硝酸中处理 $1 \mathrm{~h}$, 过后用去离子水冲洗电极 并干燥。经 $1 \mathrm{~V}$ 和 $2.05 \mathrm{~V}$ 电压沉积所制备的 GSCNTs$\mathrm{CME}$ 分别标记为电极 I 和电极 II 。

\subsection{GSCNTs/PANI-CME 和 PANI-CME 的制备}

在 $50 \mathrm{~mL}$ 含有硫酸 $(0.5 \mathrm{~mol} / \mathrm{L})$ 与苯胺 $(0.1 \mathrm{~mol} / \mathrm{L})$ 的混合溶液中通过循环伏安法(Cyclic Voltammetry, CV)制备 GSCNTs/PANI-CME: 电位范围为-0.2 1.2 V, 扫描 10 圈, 扫速为 $20 \mathrm{mV} / \mathrm{s}$ 。取出电极用二次去离 子水冲洗并在室温下干燥。1 和 $2.05 \mathrm{~V}$ 电压沉积对 应的电极分别标记为电极III和电极IV。为进行对比 实验，通过相同的 PANI 负载方法在石墨电极表面直 接电化学聚合 PANI, 获得对比电极 PANI-CME。

\section{4 表征与测试}

采用 JEOL JSM-7001F 型扫描电子显微镜(SEM, 日本日立公司)观察制备电极的形貌结构。

在 ZF-9 电化学工作站(中国上海正方公司)上进 行电化学性能测试, 采用三电极体系: 修饰电极作 为工作电极, 铂电极作为对电极, 饱和甘录电极作 为参比电极。

所用试剂均为分析纯。所用气体纯度均为 99.99\%。电化学测试所用到的 $\mathrm{pH}=6.0$ 磷酸盐缓冲 溶液(PBS)由磷酸二氢钠与磷酸氢二钠配制。

\section{2 结果与讨论}

\subsection{PANI 的电聚合}

图 1 为三种电极上电化学聚合 PANI 得到的 CV 
曲线。图 1(A)中出现了三对氧化还原峰, 峰 $\mathrm{a}$ 对应 完全还原态的 PANI 氧化为自由基阳离子的反应过 程, 峰 $\mathrm{b}$ 由 PANI 本身结构的变化(如苯醌)引起的, 峰 $\mathrm{c}$ 对应由翠绿亚胺到完全氧化态聚苯胺的转变过 程, 表明 PANI 已经成功生长在石墨电极表面 ${ }^{[30]}$ 。 随着扫描圈数的增加, 氧化还原峰峰电流随之增大, 这是因为苯胺的电聚合是一个自催化过程。同样的 现象也出现在了图 1(B)和(C)中, 只是图 1(B)和(C) 中峰电流值 $\left(I_{\mathrm{p}}\right)$ 更大。电聚合 10 圈后, 电极 $\mathrm{I}$ 中峰 $\mathrm{a}$ 的 $I_{\mathrm{p}}$ 是 $\mathrm{GE}$ 的 5 倍, 达到 $1.17 \mathrm{~mA}$; 峰 $\mathrm{b}$ 的 $I_{\mathrm{p}}$ 是 $\mathrm{GE}$ 的 5 倍, 达到 $0.85 \mathrm{~mA}$; 峰 $\mathrm{c}$ 的 $I_{\mathrm{p}}$ 是 $\mathrm{GE}$ 的 7 倍, 达 到 $1.14 \mathrm{~mA}$ 。电极 II 中峰 $\mathrm{a}$ 的 $I_{\mathrm{p}}$ 是 $\mathrm{GE}$ 的 7 倍, 达到 $1.65 \mathrm{~mA}$; 峰 $\mathrm{b}$ 的 $I_{\mathrm{p}}$ 是 $\mathrm{GE}$ 的 7 倍, 达到 $1.26 \mathrm{~mA}$; 峰 $\mathrm{c}$ 的 $I_{\mathrm{p}}$ 是 $\mathrm{GE}$ 的 10 倍, 达到 $1.7 \mathrm{~mA}$ 。因此电极 $\mathrm{I}$ 和
II 中 $I_{\mathrm{p}}$ 都远远大于 $\mathrm{GE}$ 的 $I_{\mathrm{p}}$, 这可归因于 $\mathrm{CNT}$ 优异 的电子转移速率和大的比表面积, 增强了 PANI 的 电化学性能。同时, 电极 II 中 $I_{\mathrm{p}}$ 明显大于 I 中的 $I_{\mathrm{p}}$, 说明 $2.05 \mathrm{~V}$ 沉积电压下制备的修饰电极性能更好。

\subsection{SEM 形貌分析}

图 2 为所得修饰电极的 SEM 照片。由图 2(A) 和 $(\mathrm{C})$ 可以看出，两种条件下所得 CNTs 的管壁都光 滑，且分布均匀，管状结构保存完好。但图 2(A)中 CNTs 外径在 $120 \mathrm{~nm}$ 左右, 而图 2(C)中 CNTs 的外 径却在 $50 \mathrm{~nm}$ 左右, 说明不同沉积电压会沉积不同 的镍，致使原位生长的 CNTs 的形貌(管径)出现较大 的差异; 且沉积电压越高，所得 CNTs 的管径越小。

由图 2(B)、(D)和(E)可以看出, 生长在电极表面 的 CNTs/PANI 复合材料均呈三维网状结构, 分布均
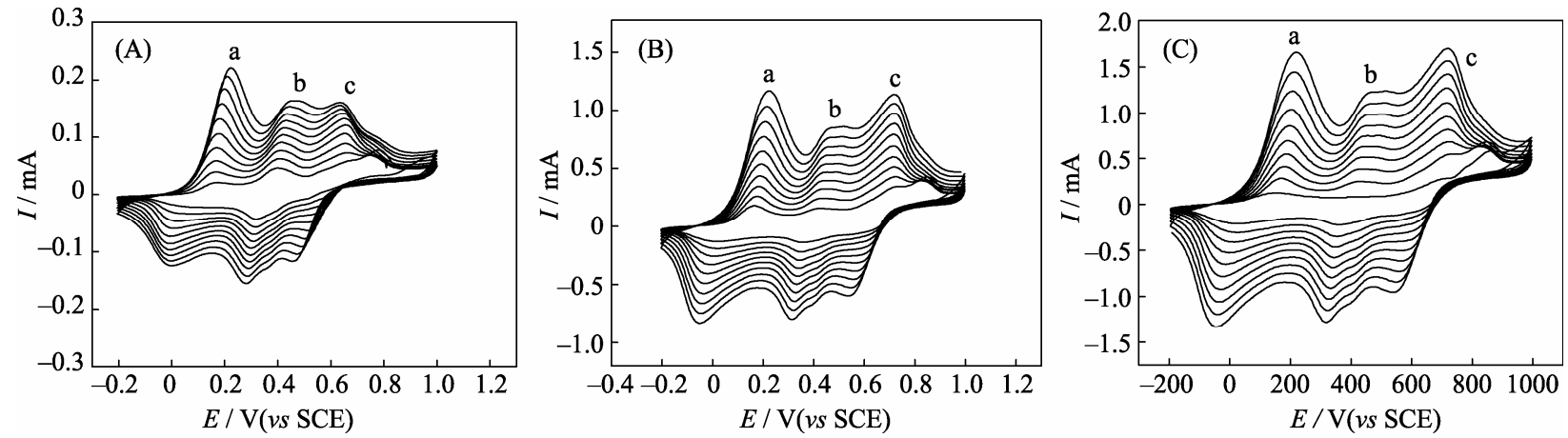

图 1 在扫速为 $20 \mathrm{mV} / \mathrm{s}$ 时, 三种电极上电化学聚合 PANI 得到的 $\mathrm{CV}$ 曲线

Fig. 1 CV curves of the PANI electrochemical polymerization on different electrodes at $20 \mathrm{mV} / \mathrm{s}$ scan rate GE (A), electrode I (B) and electrode II (C)
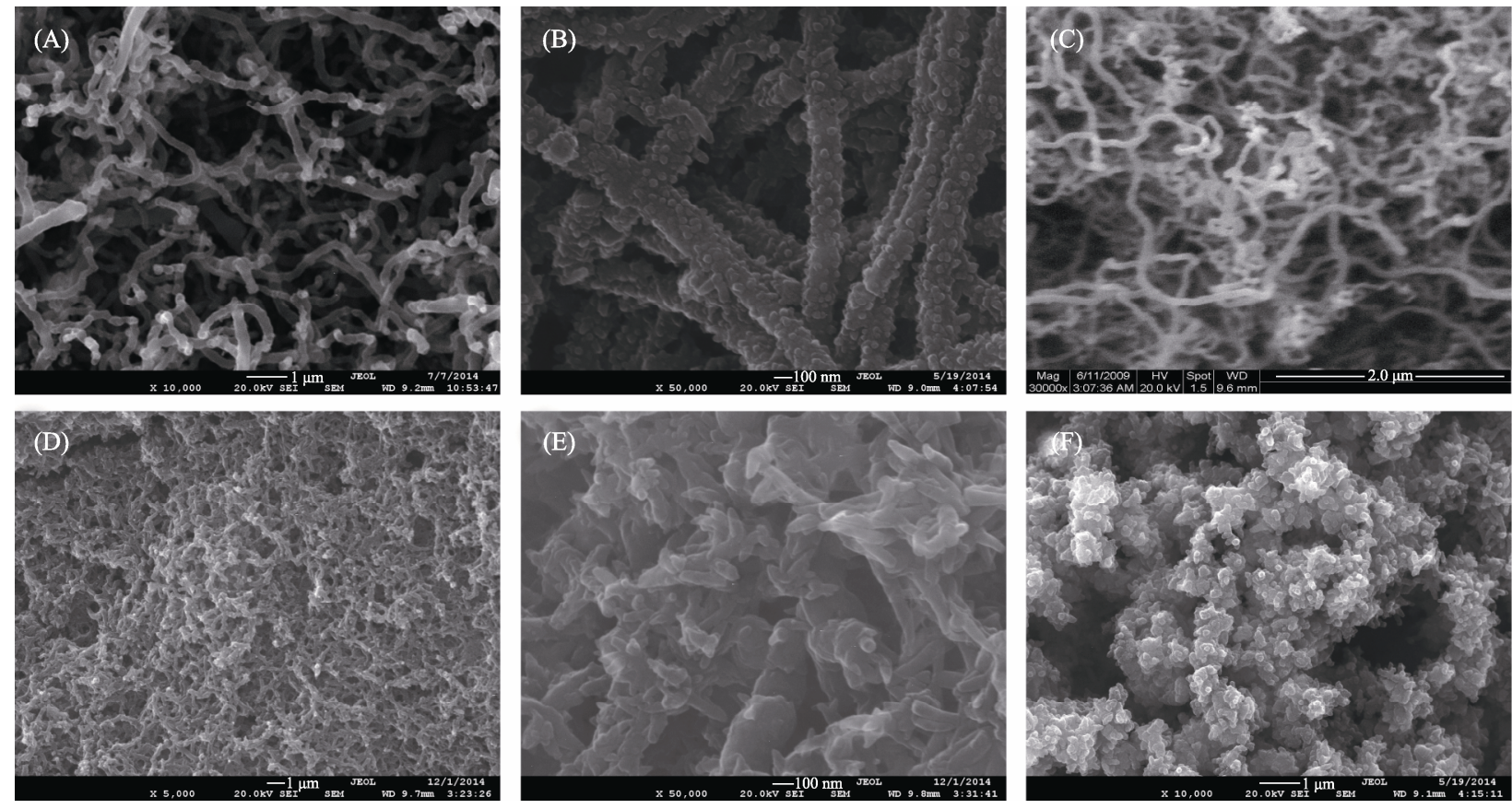

图 2 所得修饰电极的 SEM 照片

Fig. 2 SEM images of the obtained CMEs Electrode (A) I ; (B) III; (C) II ; (D, E) IV; (F) PANI-CME 
匀，管状物外壁相对于 CNTs 明显变大、变粗：图 2(B)中管状物外径在 $140 \mathrm{~nm}$ 左右; 图 2(E)中管状物 外径在 70 75 nm 之间。对比图 2(A)、(B)、(C)和(E), 可以看出电聚合 PANI 后管径明显增大，说明 PANI 已成功制备且均匀地覆盖在 CNTs 管壁上, 且在电 聚合 10 圈后，大致有 $10 \mathrm{~nm}$ 左右的厚度。对比图 2(F) 和(D)可以发现：在不存在 CNTs 时, PANI 呈颗粒状 结构, 并且相互聚集在一起; 而存在 CNTs 时, PANI 顺着 CNTs 生长, 且所得材料明显出现三维多孔结 构。在这种结构中, CNTs 起到骨架支撑作用, 发挥 高导电和高稳定性作用, 改善 PANI 的不足; 而 PANI 则充分发挥对 $\mathrm{AA}$ 的独特电化学响应能力, 共同用 于 AA 的检测。三维网状结构还有利于离子的快速 传递以及电解液的渗透, 意味着将有更好的电化学 检测性能。

\subsection{AA 在所制备电极上的电化学响应}

图 3 为所得电极在基底溶液和检测溶液中得到 的 CV 曲线图。如图 3(A) 所示, 当 AA 被添加到 PBS 溶液中时, 在 $\mathrm{CV}$ 曲线中并未出现氧化还原峰, 说 明在此实验条件下 GE 对 AA 无响应。如图 3(B)、 (C)和(D) 所示, 当 AA 添加到 PBS 溶液中时, 曲线 b 分别在 $0.35 、 0.35$ 和 $0.45 \mathrm{~V}$ 处出现明显的氧化峰(当 然也有相应的还原峰, 本文主要以氧化峰为研究对 象, 以下相同), 该氧化峰对应于 $\mathrm{AA}$ 的电化学氧化 反应， $I_{\mathrm{p}}$ 分别为 $4.5 \times 10^{-2} 、 3.6 \times 10^{-2}$ 和 $2.0 \times 10^{-2} \mathrm{~mA}$, 说明这三种修饰电极对 $\mathrm{AA}$ 的氧化具有明显的催化
作用。

如图 3(E)和(F)所示, 曲线 $\mathrm{b}$ 分别在 $0.47 \mathrm{~V}$ 、 $0.38 \mathrm{~V}$ 左右出现一个尖锐的氧化峰, $I_{\mathrm{p}}$ 分别为 $6.4 \times$ $10^{-2} 、 8.5 \times 10^{-2} \mathrm{~mA}$, 说明这两种修饰电极对 $\mathrm{AA}$ 的 电化学氧化都有较为明显的催化作用。并且相对于 图 3(B)、(C)和(D)的修饰电极而言, 这两种电极具 有更大的 $I_{\mathrm{p}}$, 说明它们对 $\mathrm{AA}$ 的电化学氧化具有更 好的电催化作用。并且电极 IV 的 $I_{\mathrm{p}}$ 高于电极III的 $I_{\mathrm{p}}$; 且电极 IV 的氧化峰峰电位 $\left(U_{\mathrm{p}}\right)$ 还低于电极III的 $U_{\mathrm{p}}$, 说明电极IV对 AA 催化作用更强。对比图 3(B) 和(C) 的结果可以发现, 两种不同沉积电压所得 CNTs$\mathrm{CME}$ 对 $\mathrm{AA}$ 的响应是不同的，电极 I 的 $I_{\mathrm{p}}$ 更大，说 明只有 CNTs 做修饰材料时, 大管径下的 CNTsCME 对 AA 的电催化更好。但对比图 3(E)和(F)的 结果可以发现, 电极 IV 对 AA 的电催化性能更好, 说明小管径的 CNTs 与 PANI 的复合，更能发挥其协 同作用，检测效果也更明显。

\section{4 修饰电极对 AA 的电化学检测的标准曲线}

图 4 为两种复合材料修饰电极分别对 $\mathrm{AA}$ 电化 学检测的标准曲线。由图 4 可知, 电极 III 在 $5.0 \times 10^{-6} \sim 4.0 \times 10^{-4} \mathrm{~mol} / \mathrm{L}$ 浓度范围内, $\mathrm{AA}$ 浓度值与 对应的 $I_{\mathrm{p}}$ 存在良好的线性关系，线性回归方程为 $I(\mu \mathrm{A})=0.309 C(\mu \mathrm{mol} / \mathrm{L})+47.176$, 线性相关系数 $r=0.99981$, 检出限为 $1.0 \times 10^{-6} \mathrm{~mol} / \mathrm{L}(\mathrm{S} / \mathrm{N}=3)$ 。而电 极 IV 则在 $1.0 \times 10^{-6} \sim 4.5 \times 10^{-4} \mathrm{~mol} / \mathrm{L}$ 浓度范围内, AA 浓度值与对应的 $I_{\mathrm{p}}$ 存在良好的线性关系，线性回归
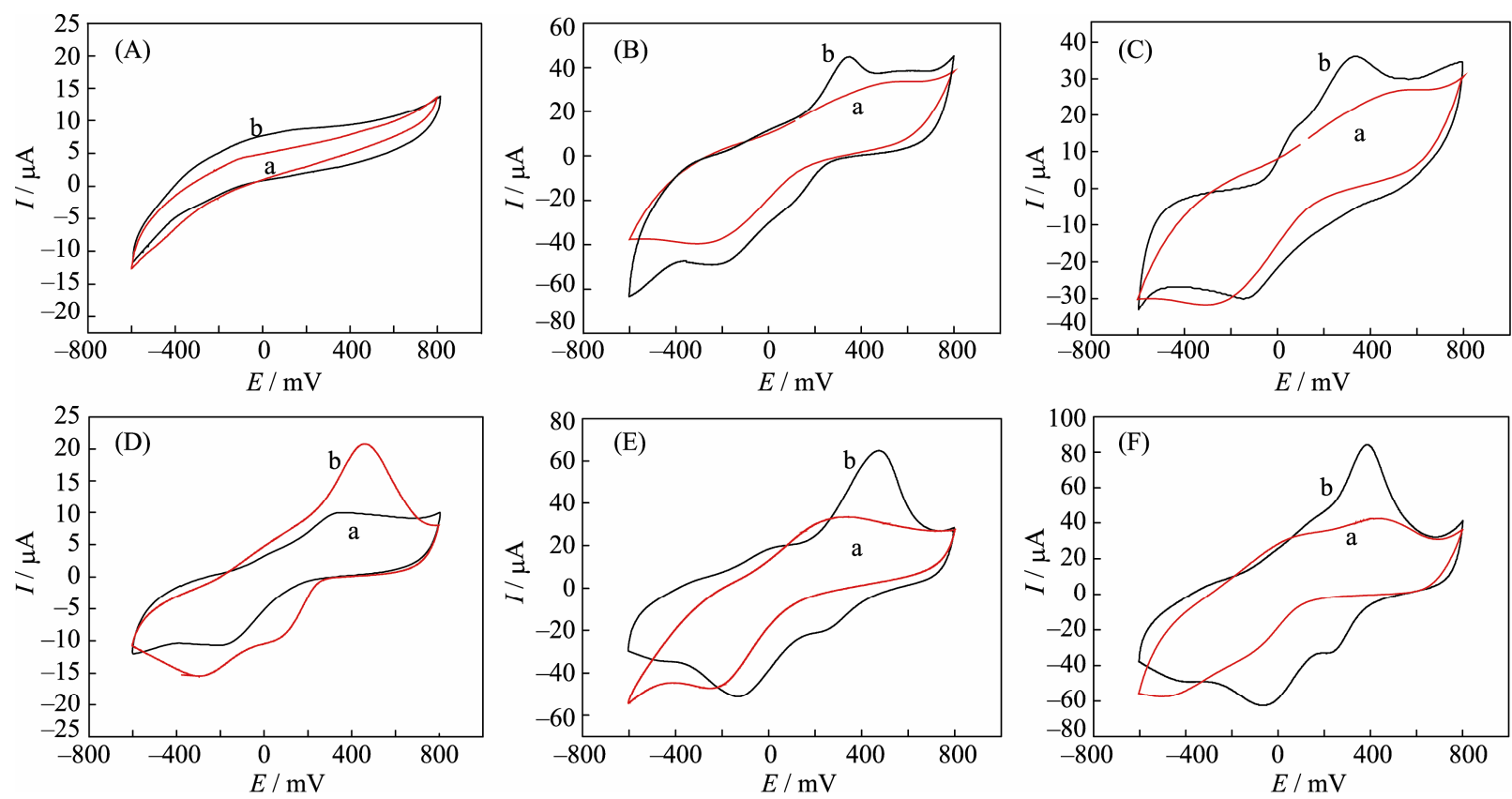

图 3 所得电极在基底溶液 $\left(\mathrm{a}, \mathrm{pH}=6.0 \mathrm{PBS}\right.$ )和检测溶液( $\mathrm{b}$, 含有 $5.0 \times 10^{-5} \mathrm{~mol} / \mathrm{L} \mathrm{AA}$ 的 $\mathrm{pH}=6.0 \mathrm{PBS}$ )中的 $\mathrm{CV}$ 曲线

Fig. $3 \mathrm{CV}$ curves of the obtained electrodes in substrate solution ( $\mathrm{a}, \mathrm{pH}=6.0 \mathrm{PBS}$ ) and detection solution ( $\mathrm{b}, \mathrm{pH}=6.0 \mathrm{PBS}$ containing $5.0 \times 10^{-5} \mathrm{~mol} / \mathrm{L} \mathrm{AA}$ ) at a scan rate of $20 \mathrm{mV} / \mathrm{s}$ Electrode: (A) GE; (B) I ; (C) II ; (D) PANI-CME; (E) III; F, IV) 
方程为 $I(\mu \mathrm{A})=0.29 C(\mu \mathrm{mol} / \mathrm{L})+70.193$, 线性相关系 数 $r=0.9992$, 检出限为 $1.0 \times 10^{-7} \mathrm{~mol} / \mathrm{L}(\mathrm{S} / \mathrm{N}=3)$ 。因 此, 电极 IV 比电极III具有更宽的线性范围和更低的 检出限, 这些数据都表示电极IV 比电极III更加优秀, 更加适合 AA 的检测。且电极IV 的检测范围与检出 限也优于其他同类电极 (见表 1)。

\section{5 电极 IV 的稳定性、重复性和可靠性实验}

电极IV 的稳定性研究是将该电极置于干燥的环 境中保存 $90 \mathrm{~d}$, 期间每十天在 $2.0 \times 10^{-4} \mathrm{~mol} / \mathrm{L} \mathrm{AA}$ PBS 溶液中做一次 CV 测试, 同时计算出每次氧化 峰峰电流值与初始值的比值, 结果如图 5(A) 所示。由 图 5(A)可知, 经过 $90 \mathrm{~d}$ 后, $\mathrm{AA}$ 的氧化峰峰电流值保
留了原有的 $92 \%$ 。此外, 将电极 $\mathrm{IV}$ 置于 $2.0 \times 10^{-4} \mathrm{~mol} / \mathrm{L}$ AA PBS 溶液中循环伏安扫描 30 次, 氧化峰峰电流值 没有明显的损失(未列出), 表现出良好的重复性 ${ }^{[35-36]}$ 。

为验证所研制电极 IV 的选择性, 进行了多巴胺 $(\mathrm{DA}) 、$ 葡萄糖(Glc)和尿酸 $(\mathrm{UA})$ 的干扰测试。在电位 $+0.4 \mathrm{~V}$ 条件下, 向 $\mathrm{pH}=6 \mathrm{PBS}$ 溶液中连续滴加 $0.2 \mathrm{mmol} / \mathrm{L} \mathrm{AA} 、 0.1 \mathrm{mmol} / \mathrm{L} \mathrm{DA} 、 0.1 \mathrm{mmol} / \mathrm{L} \mathrm{Glc}$ 、 $0.1 \mathrm{mmol} / \mathrm{L} \mathrm{UA}$ 和 $0.2 \mathrm{mmol} / \mathrm{L} \mathrm{AA}$, 结果如图 5(B)所 示。由图 5(B) 可知, 这些 $\mathrm{AA}$ 常见的干扰物对该修 饰电极和 $\mathrm{AA}$ 的响应几乎没有影响, 说明电极IV对 $\mathrm{AA}$ 具有良好的选择性和抗干扰能力。

为验证电极IV对 $\mathrm{AA}$ 测试结果的可靠性, 进行

表 1 不同修饰电极对 AA 的电化学测试性能

Table 1 Electrochemical determination performances of different electrodes for the AA

\begin{tabular}{cccc}
\hline Modified electrode & Linear range $/\left(\mathrm{mol} \cdot \mathrm{L}^{-1}\right)$ & Detection limit $/\left(\mathrm{mol} \cdot \mathrm{L}^{-1}\right)$ & Ref. \\
\hline PAn-p-aminobenzene sulfonic acid & $(3.5-17.5) \times 10^{-5}$ & $7.5 \times 10^{-6}$ & {$[31]$} \\
Molecularly imprinted PAn & $(5.0-40.0) \times 10^{-5}$ & $1.8 \times 10^{-5}$ & {$[32]$} \\
Poly (acriflavine) modified electrode & $(3.0-20.0) \times 10^{-5}$ & $1.5 \times 10^{-6}$ & {$[33]$} \\
DBSA doped Polyaniline nanoparticles & $(0.3-8.0) \times 10^{-6}$ & $8.3 \times 10^{-6}$ & {$[34]$} \\
modified electrode & & & \\
\hline
\end{tabular}
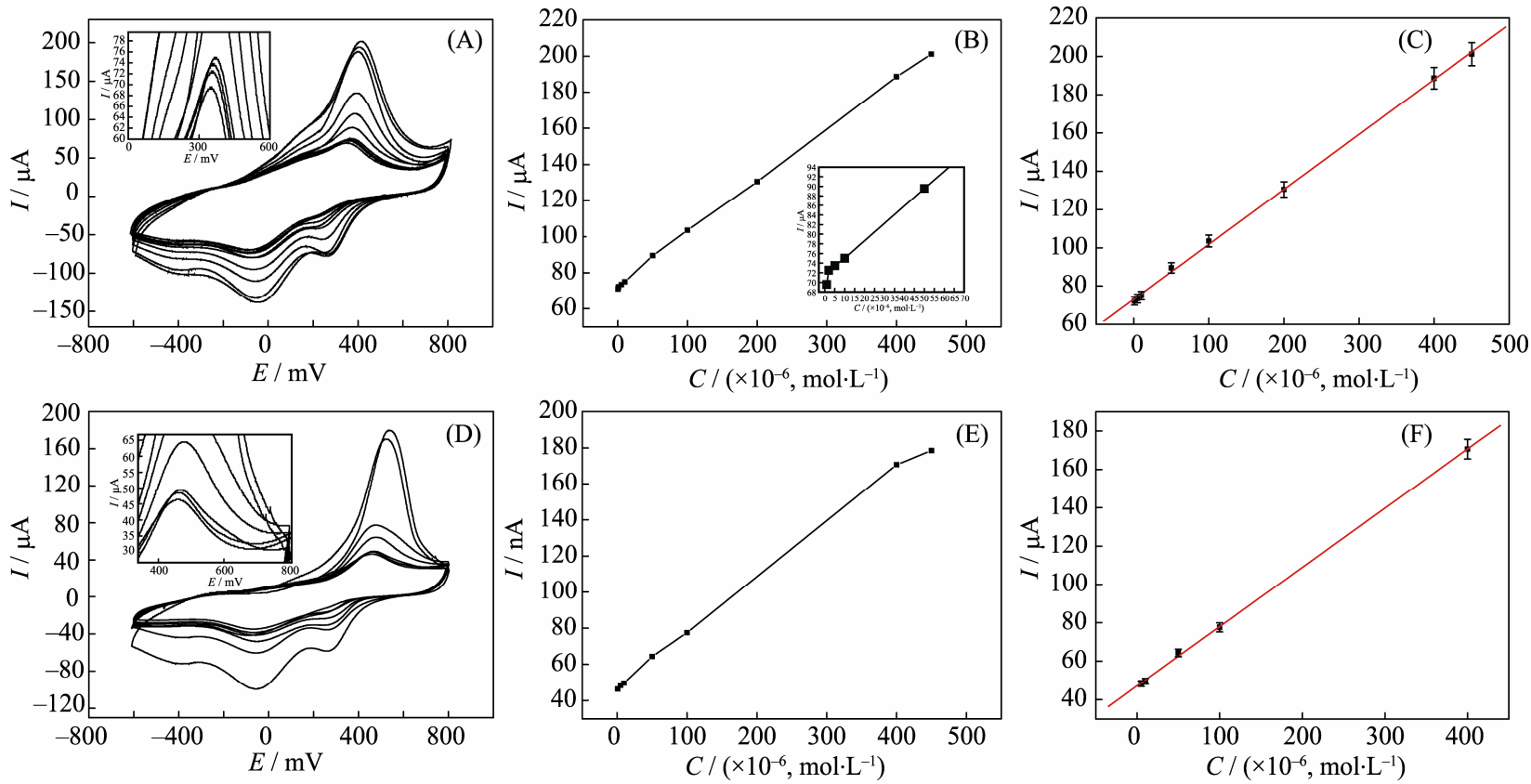

图 4 电极 IV 对 $\mathrm{AA}$ 电化学检测的标准曲线(A)不同浓度 $\mathrm{AA}$ 的 $\mathrm{CV}$ 曲线图(从内到外浓度分别为 $0.5 、 1.0 、 5.0 、 10.0 、 50.0$ 、 100.0、200.0、400.0、450.0 和 500.0 $\times 10^{-6} \mathrm{~mol} / \mathrm{L}$ ，插图为部分放大图，扫速为 $20 \mathrm{mV} / \mathrm{s}$ ); (B) AA 浓度与其对应的氧化峰峰电流 $\left(I_{\mathrm{p}}\right)$ 之间的关系图; (C) AA 浓度与其 $I_{\mathrm{p}}$ 之间的标准曲线)和电极 III对 AA 电化学检测的标准曲线; (D)不同浓度 $\mathrm{AA} C V$ 曲线图(从 内到外浓度分别为 $1.0 、 5.0 、 10.0 、 50.0 、 100.0 、 200.0 、 400.0 、 450.0 \times 10^{-6} \mathrm{~mol} / \mathrm{L}$ ，插图为部分放大图，扫速为 $\left.20 \mathrm{mV} / \mathrm{s}\right) ;(\mathrm{E}) \mathrm{AA}$ 浓度与其 $I_{\mathrm{p}}$ 之间的关系图; (F) AA 浓度与其 $I_{\mathrm{p}}$ 之间的标准曲线)

Fig. 4 Electrochemical test calibration curves of the AA based on the electrode IV ((A) CV curves at different AA concentrations: $0.5,1.0,5.0,10.0,50.0,100.0,200.0,400.0,450.0$ and $500.0 \times 10^{-6} \mathrm{~mol} / \mathrm{L}$ in $\mathrm{pH}=6.0 \mathrm{PBS}$ with $20 \mathrm{mV} / \mathrm{s}$ scan rate, the inset is an enlarged view; (B) relationship between the $I \mathrm{p}$ and AA concentrations; (C) the calibration curve of AA concentration and $I_{\mathrm{p}}$ ) and electrode III ((D) CV curves in different AA concentrations: 1.0, 5.0, 10.0, 50.0, 100.0, 200.0, 400.0 and $450.0 \times 10^{-6} \mathrm{~mol} / \mathrm{L}$ in $\mathrm{pH}=6.0$

PBS with $20 \mathrm{mV} / \mathrm{s}$ scan rate, the inset is an enlarged view; (E) relationship between the $I_{\mathrm{p}}$ and AA concentrations; (F) the calibration curve of AA concentration and $I_{\mathrm{p}}$ ) 



图 5 稳定性测试数据 $\left((\mathrm{A})\right.$ 电极IV 在含有 $2 \times 10^{-4} \mathrm{~mol} / \mathrm{L} \mathrm{AA}$ 的 $\mathrm{pH}=6 \mathrm{PBS}$ 溶液中, 扫描速率为 $20 \mathrm{mV} / \mathrm{s}$ 进行 $\mathrm{CV}$ 测试, 所 得 $I_{\mathrm{P}}$ 和储存时间的关系图)和抗干扰测试数据((B)干扰物: DA，多巴胺; Glc，葡萄糖; UA，尿酸)

Fig. 5 Data of the stability testing ((A) relationship between Ip and the storing time of the IV with $2.0 \times 10^{-4} \mathrm{~mol} / \mathrm{L}$ AA solution in $\mathrm{pH}$ 6.0 PBS at $20 \mathrm{mV} / \mathrm{s}$ scan rate) and data of the anti-jamming testing ((B) interferences: DA, dopamine; Glc, glucose; UA, uric acid))

了加标回收实验。用该电极对样品进行检测, 测得 样品中 $\mathrm{AA}$ 的浓度为 $1.054 \times 10^{-5} \mathrm{~mol} / \mathrm{L}(\mathrm{RSD}=1.9 \%)$ 。 然后, 将不同浓度的 $\mathrm{AA}$ 添加到样品中进行回收实 验, 实验结果如表 2 所示。由表 2 可知, 回收率在 97.4\%和 101.2\%之间, 同时每个浓度三个平行测试 得到的 RSD 小于 $2.5 \%$, 表明电极 IV 对 AA 的检测结 果具有良好的可靠性。

\section{3 结论}

1) 通过 CNTs 在石墨电极表面的原位生长以及

\section{表 2 所得电极对 AA 的回收实验数据}

Table 2 Determination of AA in the recovery experiments

\begin{tabular}{ccccrc}
\hline Samples & $\begin{array}{c}\text { Detected } \\
\left(\times 10^{-6},\right. \\
\left.\mathrm{mol} \cdot \mathrm{L}^{-1}\right)\end{array}$ & $\begin{array}{c}\text { Added } \\
\left(\times 10^{-6},\right. \\
\left.\mathrm{mol} \cdot \mathrm{L}^{-1}\right)\end{array}$ & $\begin{array}{c}\text { Found } \\
\left(\times 10^{-6},\right. \\
\left.\mathrm{mol} \cdot \mathrm{L}^{-1}\right)\end{array}$ & $\begin{array}{c}\text { Recov- } \\
\text { ery } / \%\end{array}$ & $\begin{array}{c}\mathrm{RSD} / \\
(\%, \\
n=6)\end{array}$ \\
\hline 1 & & 20.00 & 30.02 & 97.4 & 2.5 \\
2 & \multirow{2}{*}{10.54} & 40.00 & 51.37 & 102.1 & 1.9 \\
3 & & 60.00 & 69.86 & 98.9 & 0.8 \\
4 & & 80.00 & 91.48 & 101.2 & 1.0 \\
\hline
\end{tabular}

PANI 的原位电聚合, 成功制备了 GSCNTs/PANICME。且 CNTs 在石墨电极表面生长均匀, 保持完 整的管状结构, PANI 均匀地包覆在 CNTs 管壁上, 生长在电极表面的复合材料呈现明显的三维网状 结构。

2) CNTs 的管径可以通过恒电压沉积催化剂镍 的电压大小来控制，且 CNTs 管径的大小对 GSCNTs/ PANI-CME 电化学检测性能有着重要的影响。具有 较小 CNTs 管径的 GSCNTs/PANI-CME 对 AA 具有 更加优良的检测性能。

3) GSCNTs/PANI-CME 在 $1.0 \times 10^{-6} \mathrm{~mol} / \mathrm{L}$ 至 $4.5 \times$ $10^{-4} \mathrm{~mol} / \mathrm{L}$ 浓度范围内, 氧化峰峰电流与 $\mathrm{AA}$ 浓度呈 良好的线性关系，线性回归方程为 $I(\mu \mathrm{A})=0.29 \mathrm{C}$ $(\mu \mathrm{mol} / \mathrm{L})+70.193$, 检出限为 $1.0 \times 10^{-7} \mathrm{~mol} / \mathrm{L}(\mathrm{S} / \mathrm{N}=$ 3)。且检测性能不受常见干扰物的影响，所制备修 饰电极具有高的重复性与稳定性。

\section{参考文献:}

[1] WU G, WU Y, LIU X, et al. An electrochemical ascorbic acid sensor based on palladium nanoparticles supported on graphene oxide. Analytica Chimica Acta, 2012, 745: 33-37.

[2] XI L, REN D, LUO J, et al. Electrochemical analysis of ascorbic acid using copper nanoparticles/polyaniline modified glassy carbon electrode. Journal of Electroanalytical Chemistry, 2010, 650(1): 127-134.

[3] DONG Y P, HUANG L, ZhANG J, et al. Electro-oxidation of ascorbic acid at bismuth sulfide nanorod modified glassy carbon electrode. Electrochimica Acta, 2012, 74: 189-193.

[4] ZHANG Y J, WEN Y, LIU Y, et al. Functionalization of single-walled carbon nanotubes with Prussian blue. Electrochemistry Communications, 2004, 6: 1180-1184.

[5] WANG Y, LI Y M, TANG L H, et al. Application of graphene-modified electrode for selective detection of dopamine. Electrochemistry Communications, 2009, 11: 889-892.

[6] ZUO X, ZHANG H, LI N. An electrochemical biosensor for determination of ascorbic acid by cobalt (II) phthalocyanine- multi-walled carbon nanotubes modified glassy carbon electrode. Sensors and Actuators B: Chemical, 2012, 161(1): 1074-1079.

[7] LI F, TANG C, LIU S, et al. Development of an electrochemical ascorbic acid sensor based on the incorporation of a ferricyanide mediator with a polyelectrolyte-calcium carbonate microsphere. Electrochimica Acta, 2010, 55(3): 838-843.

[8] ZHANG L, SHI H W, WANG C, et al. Preparation of a nanocomposite film from poly(diallydimethyl ammonium chloride) and gold nanoparticles by in-situ electrochemical reduction, and its application to SERS spectroscopy and sensing of ascorbic acid. Microchimica Acta, 2011, 173(3/4): 401-406.

[9] XI L, REN D, LUO J, et al. Electrochemical analysis of ascorbic acid using copper nanoparticles/polyaniline modified glassy carbon electrode. Journal of Electroanalytical Chemistry, 2010, 650(1): 127-134.

[10] RANA U, PAUL N D, MONDAL S, et al. Water soluble polyaniline coated electrode: a simple and nimble electrochemical approach for ascorbic acid detection. Synthetic Metals, 2014, 192: 43-49. 
[11] IIJIMA S. Helical microtubules of graphitic carbon. Nature, 1991, 354(6348): 56 .

[12] MUSAMEH M, WANG J, MERKOCI A, et al. Low-potential stable NADH detection at carbon-nanotube-modified glassy carbon electrodes. Electrochemistry Communications, 2002, 4(10): 743746.

[13] DEO R P, WANG J. Electrochemical detection of carbohydrates at carbon-nanotube modified glassy-carbon electrodes. Electrochemistry Communications, 2004, 6(3): 284-287.

[14] DONG S, ZHANG S, CHI L, et al. Electrochemical behaviors of amino acids at multiwall carbon nanotubes and $\mathrm{Cu}_{2} \mathrm{O}$ modified carbon paste electrode. Analytical Biochemistry, 2008, 381(2): 199-204.

[15] ZIYATDINOVA G, ZIGANSHINA E, BUDNIKOV H. Electrooxidation of morin on glassy carbon electrode modified by carboxylated single-walled carbon nanotubes and surfactants. Electrochimica Acta, 2014, 145: 209-216.

[16] LI Y, UMASANKAR Y, CHEN S M. Polyaniline and poly (flavin adenine dinucleotide) doped multi-walled carbon nanotubes for p-acetamidophenol sensor. Talanta, 2009, 79(2): 486-492.

[17] YUN J, IM J S, KIM H I, et al. Effect of oxyfluorination on gas sensing behavior of polyaniline-coated multi-walled carbon nanotubes. Applied Surface Science, 2012, 258(8): 3462-3468.

[18] ZOU Y, SUN L X, XU F. Biosensor based on polyaniline-Prussian Blue/multi-walled carbon nanotubes hybrid composites. Biosensors and Bioelectronics, 2007, 22(11): 2669-2674.

[19] YANG T, ZHOU N, ZHANG Y, et al. Synergistically improved sensitivity for the detection of specific DNA sequences using polyaniline nanofibers and multi-walled carbon nanotubes composites. Biosensors and Bioelectronics, 2009, 24(7): 2165-2170.

[20] MANISANKAR P, SUNDARI P L A, SASIKUMAR R, et al. Electroanalysis of some common pesticides using conducting polymer/multiwalled carbon nanotubes modified glassy carbon electrode. Talanta, 2008, 76(5): 1022-1028.

[21] PILAN L, RAICOPOL M. Highly selective and stable glucose biosensors based on polyaniline/carbon nanotubes composites. Journal of Scientific Bulletin, 2014, 76(1): 155-166.

[22] LUO X, KILLARD A J, MORRIN A, et al. Enhancement of a conducting polymer-based biosensor using carbon nanotube-doped polyaniline. Analytica Chimica Acta, 2006, 575(1): 39-44.

[23] XI L, ZHU Z, WANG F. Electrocatalytic oxidation of ascorbic acid on quaternized carbon nanotubes/ionic liquid-polyaniline composite film modified glassy carbon electrode. Journal of the Electrochemical Society, 2013, 160(6): H327-H334.

[24] CHAWLA S, RAWAL R, SHARMA S, et al. An amperometric biosensor based on laccase immobilized onto nickel nanoparticles/ carboxylated multiwalled carbon nanotubes/polyaniline modified gold electrode for determination of phenolic content in fruit juices.
Biochemical Engineering Journal, 2012, 68: 76-84.

[25] FENG X, LI R, MA Y, et al. The synthesis of highly electroactive $\mathrm{N}$-doped carbon nanotube/polyaniline/Au nanocomposites and their application to the biosensor. Synthetic Metals, 2011, 161(17): 1940-1945.

[26] DING L, LI Q, ZHOU D, et al. Modification of glassy carbon electrode with polyaniline/multi-walled carbon nanotubes composite: application to electro-reduction of bromate. Journal of Electroanalytical Chemistry, 2012, 668: 44-50.

[27] XU L, ZHU Y, YANG X, et al. Amperometric biosensor based on carbon nanotubes coated with polyaniline/dendrimer-encapsulated Pt nanoparticles for glucose detection. Materials Science and Engineering: $C$, 2009, 29(4): 1306-1310.

[28] JIANG Q, YANG R, HE Z, et al. Preparation and characterization of a graphite electrode containing carbon nanotubes grown in situ by flame synthesis. Electrochimica Acta, 2011, 56(14): 52055209.

[29] JIANG Q, SONG L J, YANG H, et al. Preparation and characterization on the carbon nanotube chemically modified electrode grown in situ. Electrochemistry Communications, 2008, 10(3): 424-427.

[30] MANESH K M, SANTHOSH P, KOMATHI S, et al. Electrochemical detection of celecoxib at a polyaniline grafted multiwall carbon nanotubes modified electrode. Analytica Chimica Acta, 2008, 626(1): 1-9.

[31] ZHANG L, ZHANG C, LIAN J. Electrochemical synthesis of polyaniline nano-networks on p-aminobenzene sulfonic acid functionalized glassy carbon electrode: its use for the simultaneous determination of ascorbic acid and uric acid. Biosensors and Bioelectronics, 2008, 24(4): 690-695.

[32] ROY A K, DHAND C, MALHOTRA B D. Molecularly imprinted polyaniline film for ascorbic acid detection. Journal of Molecular Recognition, 2011, 24(4): 700-706.

[33] NIEN P C, CHEN P Y, HO K C. On the amperometric detection and electrocatalytic analysis of ascorbic acid and dopamine using a poly (acriflavine)-modified electrode. Sensors and Actuators B: Chemical, 2009, 140(1): 58-64.

[34] AMBROSI A, MORRIN A, SMYTH M R, et al. The application of conducting polymer nanoparticle electrodes to the sensing of ascorbic acid. Analytica Chimica Acta, 2008, 609(1): 37-43.

[35] DEVI R, YADAV S, PUNDIR C S. Electrochemical detection of xanthine in fish meat by xanthine oxidase immobilized on carboxylated multiwalled carbon nanotubes/polyaniline composite film. Biochemical Engineering Journal, 2011, 58: 148-153.

[36] ZHONG H, YUAN R, CHAI Y, et al. In situ chemo-synthesized multi-wall carbon nanotube-conductive polyaniline nanocomposites: characterization and application for a glucose amperometric biosensor. Talanta, 2011, 85(1): 104-111. 